\title{
Erratum to: Quality control verification and mapping for chemical application
}

\author{
D. K. Giles • D. Downey
}

Published online: 7 May 2010

(C) Springer Science+Business Media, LLC 2010

\section{Erratum to: Precision Agric (2003) 4:103-124 DOI: $10.1023 / \mathrm{A}: 1021871207195$}

Recent re-evaluation of the original work published in 2003 found that theoretical droplet size diameters were reported incorrectly. In all cases reported in the original work, the theoretical droplet diameters were under-reported by $17.5 \%$. The error in theoretical droplet diameter resulted in an incorrect estimate for spread factor (reported as 0.392 in the original work). The correct spread factor, based on the ratio of the "correct" theoretical droplet diameter to the measured stain diameter is 0.474 . In all cases, the droplet size distributions were actually $17.2 \%$ higher than what was originally reported. It should be noted that the conclusions from the original work are unchanged, viz., blended-pulse control during large-scale spray applications provides a wider dynamic range and better control for maintaining consistency of spray deposition. Results presented in the original work showed that droplet size changes for mitigation of spray drift were possible while sensing weather conditions and potentially hazardous sprayer locations. Additionally, use of on-board GPS and environmental data collection equipment for mapping application parameters and environmental conditions can assist applicators in providing efficacious and safe applications while also providing documentation of the application for regulatory compliance.

The online version of the original article can be found under doi:10.1023/A:1021871207195.

D. K. Giles · D. Downey $(\bowtie)$

Department of Biological and Agricultural Engineering, University of California-Davis, Davis, CA 95616, USA

e-mail: ddowney@ucdavis.edu 